(2) Open Access Full Text Article

ORIGINAL RESEARCH

\title{
Attention Impairment During the Interictal State in Migraineurs without Aura: A Cross-Sectional
} Study

\author{
Cheng Chen $\mathbb{1}^{1,2, *}$ \\ Xin Dong $\mathbb{D}^{1, *}$ \\ Ping Gu (D) \\ Keyan Chen (1D) \\ Qi Wan (D)' \\ Haiyue Xie $\mathbb{D i D}^{3}$ \\ Zhaochun Shi $\mathbb{D}^{\prime}$ \\ Teng Wang'
}

'Department of Neurology, The First Affiliated Hospital of Nanjing Medical University, Nanjing, Jiangsu, 210029 ,

People's Republic of China; ${ }^{2}$ Department of Neurology, The Second Affiliated Hospital of Wannan Medical College, Wuhu, Anhui, 241000, People's Republic of China; ${ }^{3}$ The First Clinical Medical College of Nanjing Medical University, Nanjing, Jiangsu, 210029, People's

Republic of China

*These authors contributed equally to this work
Background and Purpose: Migraine suffering is more than the onset of head pain. The broad non-painful clinical symptoms associated with migraine are not well recognized. Recent researches support that migraineurs suffer attention deficits, but these findings are not conclusive. The purpose of our study was to assess whether patients with migraine without aura (MwoA) during the interictal period have attention impairment and to identify the migraine characteristics related to attention deficits.

Methods: We enrolled subjects with MwoA during the interictal period and healthy controls matched for age, gender, and education level in this cross-sectional study. The attention network test (ANT) and a battery of neuropsychological tests, including the trail-making test (TMT), the digit span test (DST), and the Stroop test, were administered to the participants during the headache-free period.

Results: Forty-four subjects with MwoA (4 males, 40 females) and 20 controls matched for age, gender, and literacy education were included. Patients in MwoA were more anxious $(P=$ $0.007)$ and depressed $(P=0.001)$ than healthy subjects. Significant differences between the two groups were detected in the executive network $(P=0.006)$ but not in the alerting and orienting networks of ANT. Mean reaction time of ANT in the MwoA group was significantly longer than that in the control group $(P=0.028)$. Patients showed worse performance on DST-forward $(P<0.001)$, DST-backward $(P<0.001)$, DS Total $(P<0.001)$, TMT-A $(P<$ $0.001)$, TMT-B $(P<0.001)$ and TMT-d $(P=0.002)$. Differences found in executive functions between the two groups were unrelated to gender, age, literacy, anxiety, and depression. Multiple regression analysis revealed no relation between clinical characteristics of headache and scores on the executive function with MwoA.

Conclusion: Our study suggested that patients in MwoA present worse performances on the executive control of attention networks during the headache-free period, which appear not be associated with measures of migraine severity. Although more studies are needed in this area, our results could be useful to find specific neuropsychological biomarker for migraine pathophysiology.

Keywords: migraine without aura, attention, attention network test, executive function, reaction time

\section{Introduction}

Migraine is regarded as a complex brain disorder and characterized by unilateral or bilateral, moderate to severe pulsatile headache with a variety of associated symptoms. $^{1,2}$ In the Global Burden of Disease Study 2016, migraine is reported as the second most disabling disorder affecting $9 \%$ to $35 \%$ of the world population,

\footnotetext{
Correspondence: Teng Wang; Zhaochun Department of Neurology, The First Affiliated Hospital of Nanjing Medical University, Nanjing, Jiangsu, 210029 ,

People's Republic of China

Tel +86256830 6050; +8625683036I3

Fax +86 2583718836

Email tengwnjmu@I63.com;

290694748@qq.com
} 
most of whom are at the highest economically productive age. ${ }^{3}$ In addition to pain, which is the main determinant of disability, cognition impairment as the non-core symptoms usually bothered migraineurs and contributed to their poor quality of health-related life during and even between migraine attacks. ${ }^{4}$ Recently, a growing body of studies have attracted much attention to the cognitive performance in migraineurs. ${ }^{5-8}$

Attention is defined as a basic cognitive function that ensures the correct allocation of processing resources to the relevant stimuli. ${ }^{9}$ It is considered as one of the specific cognitive domains and predominantly affects migraine sufferers. ${ }^{10}$ Subjective attention impairment was frequently reported by migraineurs, such as lower ability to concentrate, difficulty thinking, losing the notion of things. ${ }^{11}$ Previous studies have imposed a consistent view of impaired attention in the migraine attack period. ${ }^{12,13}$ However, scant consistent information is available on attention deficits in patients with migraine without aura during the interictal period. The divergent results in the interictal period may be due to definitions of attention and the application of qualitatively different instruments to measure attention.

Advances in cognitive neuroscience have evidenced that the human attentional system is viewed as the brain network function involving three independent subsystems (alerting, orienting, and executive control), which are served by anatomically distinct brain regions and innervated by different neuromodulators. ${ }^{14,15}$ The attention network test (ANT), first proposed by Fan et $\mathrm{al}^{15}$ is a computerized neuropsychological test and has been validated as a simple and reliable way to assess the processing efficiency of the three separate attention networks. In recent years, ANT has been applied to investigate attention in various neurological diseases such as Parkinson's disease, ${ }^{16}$ Attention Deficit Hyperactivity Disorder, ${ }^{17}$ and Multiple Sclerosis. ${ }^{18}$ However, limited information is available on the ANT applied in patients with migraine. Based on the above considerations, our cross-sectional study aimed to assess the attention function in migraineurs without aura during the interictal period using the ANT and a series of attention scales and to identify the migraine characteristics related to attention deficits.

\section{Methods}

\section{Patients}

Our study recruited 44 patients with migraine without aura (MwoA) consecutively visiting the neurology clinic in the
First Affiliated Hospital of Nanjing Medical University between August 2018 and December 2019. Twenty healthy controls (HC) without a history of migraine or familial aggregation for migraine were recruited from patients' friends, employees at the clinic or university centers. All groups' enrollment was performed by two neurologists, specialist in headache disorders according to the International Classification of Headache Disorders, third edition (ICHDIII) ${ }^{19}$ at their first visits. The inclusion criteria for patients as follows: a) at least one attack per month; b) the duration of migraine history was at least more than one year; c) all migraineurs were in the interictal period when they were tested (at least four days after the last headache attack and free of attacks two days after the evaluation); d) Patients were not receiving prophylactic therapy and medicine-free for at least $24 \mathrm{~h}$. All participants, aged 18 to 65, were right-handed, had normal or corrected to normal vision and hearing, had at least five years of formal education, and no dementia. Subjects with other headache types, brain injuries, psychiatric, neurodegenerative disease, a history of alcohol or drug abuse, medication affecting cognition or any chronic conditions requiring daily treatment were excluded from our study. The procedure of our observational study was approved by the First Affiliated Hospital of Nanjing Medical University Ethics Committee (2017-SR-046). All subjects signed written informed consent in agreement with the principles of the Declaration of Helsinki.

\section{Migraine Characteristics}

The clinical data collection at baselines such as gender, age, educational level, the length of migraine history, number of headache days per month, the score of pain intensity, current treatments, and extensive physical examination was performed at the first visit by the headache specialists. The impact of migraine disorder was assessed with the Migraine-Specific Quality of Life Questionnaire (MSQ). Pain intensity represented the average intensity of headache attacks experienced in the last three months was scored with a 10-point visual analog scale (VAS). Migraine days per month were calculated as the mean days (per month) of the migraine attacks in the previous three months. These data were obtained using a structural headache questionnaire and self-reports based on the ICHD-III criteria for migraine. ${ }^{19}$

\section{Neuropsychological Scale Tests}

Neuropsychological tests were performed by a trained headache specialist who was blinded to patients' identity. 
The Chinese version of the Mini-Mental State Examination (MMSE-C) was used for excluding dementia patients. Self-Rating Depression Scale (SDS) and SelfRating Anxiety Scale (SAS) were used to assess the degree of depression and anxiety. The neuropsychological scale adopted in our study to evaluate the attention function included the Trail-Making Test (TMT), the Digit Span test (DST), the Stroop test.

\section{Chinese Version of the Trail-Making Test (TMT)}

The Chinese version of TMT comprises two components (A and B). In TMT-A, participants were instructed to connect the 25 encircled numbers arranged randomly on an A4 page $(21 \times 29 \mathrm{~cm})$ in ascending order as rapidly as possible (ie, (1) - (2) - (3)-, etc.). The test would be terminated if the duration exceeds $150 \mathrm{~s}$. The TMT-B required individuals to connect randomly arranged numbers with a line, alternating between circles and squares (ie, (1)- 1 -2)- -2- -3)- -3, etc.) as rapidly as possible on the same size paper. A maximum time of 300 seconds was allowed for completion. The time (in seconds) taken to complete each part of the task (A and B) was recorded as the direct scores of TMT. TMT-A was used for processing speed, while TMT-B was related to attentional set-shifting. The TMT-d (TMT difference) score was calculated from the TMT-B score minus the TMT-A score, which could better reflect the efficiency of executive functions. ${ }^{20}$

\section{Digit Span Test (DST)}

Two subtests (digit forward and digit backward) are involved in this test. The digit forward test (DST-f) seems to be related mostly to immediate auditory memory while the digit backward test (DST-b) is likely to involve working memory and mental flexibility. In the digit forward test (DST-f), subjects were instructed to repeat the random number sequences that become gradually longer, in the order with that the examiner read. By contrast, in the digit backward test (DST-b), the participants were required to recall a series of digits that become gradually longer, in the reverse order, with that the examiner read. The forward (DST-f) and backward (DST-b) scores were the maximum digit span that the participants were able to repeat in direct and reverse orders, respectively. The Digit Span (DS) total was the combined raw scores of DST-f and DST-b. ${ }^{21}$

\section{Stroop Test}

The Stroop test contains three-part tasks, which is widely used to test processing speed and cognitive flexibility, and is considered as a reliable tool for the evaluation of executive function. Part A task was the "Word Reading" (WR) task in which the subject was required to read the list of 100 color names ("Yellow", "Red", "Green", and "Blue") printed in black ink with Chinese characters on the card A. Part B task was the "Color Naming" $(\mathrm{CN})$ task in which the individual was asked to name the color for each circle in the list of 100 circles printed with different colors on the card B. The last part C task was the "Color Word" (CW) test in which the participant must name the ink color and disregard the verbal content of the written Chinese characters expressing different colors. The direct score of each task was the number of items completed in the given time (45 seconds). The interference score (IS) was calculated with the formula: $\mathrm{IS}=\mathrm{CW}-(\mathrm{WR} * \mathrm{CN}) /(\mathrm{WR}+\mathrm{CN}){ }^{22}$

\section{Attention Network Test Procedure: The Attention Network Test}

The ANT used in this study is the original version, first proposed by Fan et al. ${ }^{15}$ The total of ANT consisted of four sessions: one practice test and three formal tests (Figure 1A). The practice test contained 24 trials, which was used to check whether the patients have grasped the operation process, while 96 trials were included in each formal test, and each trial took 4000ms (four cue types $\times$ two target locations $\times$ two target directions $\times$ three congruencies $\times$ two repetitions). Participants could have a break for one minute between test sessions. The detailed process of each trial in ANT was illustrated as follows (Figure 1B). In each session, participants were required to stare at a fixation cross in the computer screen center during the test and to identify the central arrow direction of the target stimuli according to the warning cue as soon as possible. The mode of cue and target stimuli appeared randomly. Subjects' response data based on the target stimuli were recorded via pressing the left or right arrow keys on the keyboard. Each trial was initiated with a fixation cross shown in the center of the screen lasted for a random variable duration (400 to $1600 \mathrm{~ms}$ ). Then, one of the four cue types (no cue, center cue, double cue, or spatial cue) was rendered for $100 \mathrm{~ms}$ on the screen. In no cue condition, there was no asterisk precedes the target stimuli. According to the position of the asterisk relative to the fixation cross, the warning cue could be either a central cue (the asterisk 

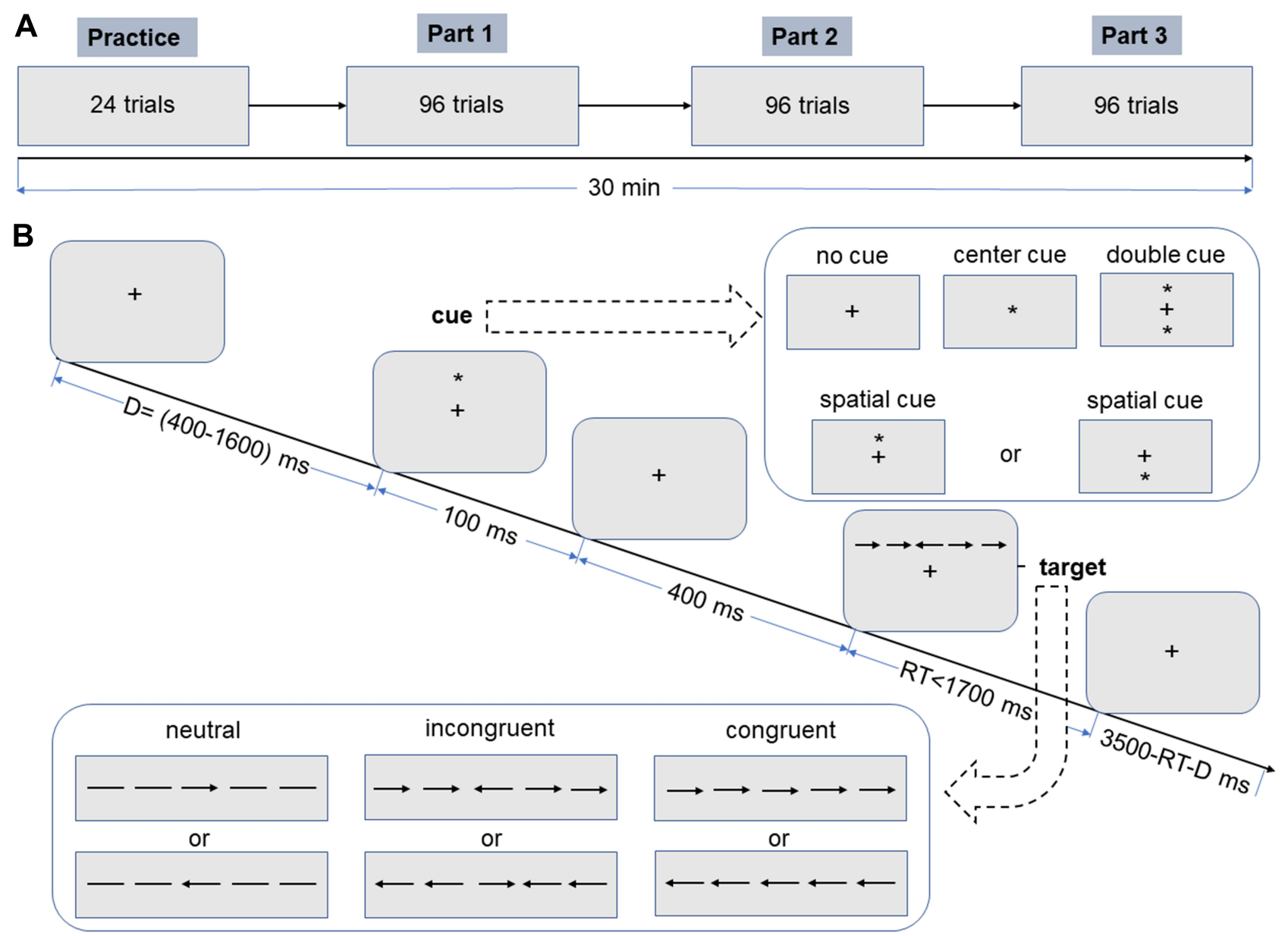

Figure I The experimental paradigm of the attention network test. (A) Four sessions in the ANT. (B) The timeline of each trial used in each session.

replaces the fixation in the center of the screen) or a spatial cue (the asterisk is displayed above or below the fixation cross where the target would later present), or double cue (two asterisks, respectively, appear above and below the fixation cross, both of which are likely to be where the target would appear). These cues provided temporal information about the coming target stimuli, and all spatial cues provided valid information indicating the location the arrow would appear exactly. After the cue presentation, a fixation cross was presented for $400 \mathrm{~ms}$ in the screen center again before the target stimuli. The subsequent target stimuli required participants to provide a fast and accurate response in recognizing the left or right directivity of the central arrow accompanied on each side by two flankers (either arrows or lines) on the screen in no more than $1700 \mathrm{~ms}$ according to the warning cue. The four flankers could be either line without arrowheads (neutral condition), or arrows pointing in the same direction as the central arrow (congruent condition), or arrows in the opposite direction to the central arrow (incongruent condition). At the end of the trial, a fixation cross was kept visible in the center of the screen with the duration depending on the first fixation and the reaction time (RT).

\section{ANT Data Analysis}

The accuracy and reaction time recordings for different conditions were recorded by E-prime software 2.0. The overall mean reaction time (RT) for each subject was obtained from the average reaction time for 12 combinations of the four cue conditions (no cue, central cue, double cue, spatial cue) and the three flanker conditions (congruent, incongruent, neutral). Accuracy was calculated from the proportion of correct responses in all trials. The efficiency of the alerting, orientating, and executive control networks were defined as the mean reaction time difference calculated by subtracting RTs under different conditions, which indicated how RT was influenced by alerting cues (no cue versus double cue), spatial cues 
(center cue versus spatial cue), and flankers (congruent versus incongruent). The formulas were as follow: 1) alert network efficiency $=$ mean RT (no cue) - mean RT (double cue); 2) orient network efficiency $=$ mean RT (center cue) - mean RT (spatial cue); 3) executive network efficiency $=$ mean RT (incongruent) - mean RT (congruent). The higher value of RT subtraction for the alert network and orient network manifested the greater efficiency. Conversely, the higher value of RT subtraction for the executive control network indicated the lower efficiency.

\section{Statistical Analysis}

Kolmogorov-Smirnov test was employed to check the assumption of normality for the research variables. Student's $t$-test for the normally distributed variables and the Mann-Whitney $U$-test for the skewed distribution data were used to compare variables between migraine patients and normal controls. The Chi-square test was used to compare the distribution by gender. To satisfy the normality assumption for statistical analyses, the arithmetic transformation was applied to "TMT-d" and "Accuracy". The two dependent variables ("RT" and "Accuracy") were analyzed by means of the mixed model analysis of variance (ANOVA) for repeated measures, with "cue" (no cue, double cue, center cue, and spatial cue) and "flanker" (congruent, incongruent, and neutral) as the within-subject factors, and "group" (MwoA and control) as the betweensubject factor. Multiple regression analysis was calculated to assess the effect of the following variables on the identified difference: gender, age, literacy, anxiety, and depression. The statistical analysis was performed by SPSS 23.0 (SPSS Inc., Chicago, Illinois, USA). Significant levels were set at $P<0.05$.

\section{Results}

\section{Demographic and Migraine Characteristics}

Fifty consecutive patients with MwoA were screened. Only 44 migraineurs without aura (40 females and 4 males) were enrolled since three patients experienced migraine attacks during the neuropsychological assessment, and three patients dropped out of the procedure. Twenty healthy controls were included in our study. Demographic and migraine characteristics are shown in Table 1. In our study, the mean headache days migraineurs suffered per month were 9 (range 4 to 20) days. On average, migraine participants had been suffering headaches for $(17.36 \pm 10.55)$ years. The pain intensity migraineurs reported was 7.5 (range 7.00 to 8.38 ) by VAS. The mean MSQ scores were $40.91 \pm 9.74$. There were no differences in age, sex ratio, and education level between the cases and controls. Subjects with MwoA reported significantly higher levels of anxiety $(P=0.007)$ and depression $(P=0.001)$ as compared with controls.

\section{ANT}

\section{RT and Accuracy}

The RT and accuracy values in ANT for each group are summarized in Table 2. ANOVA for RTs revealed the significant main effect of group, cue condition, and flanker type $(F=84.085, P<0.001 ; F=10.016, P<0.001 ; F=138.008$, $P<0.001$, respectively). Significant "group" $\times$ "flanker" interaction $(F=4.436, P=0.012)$ was observed. There was no significant interaction effect of "group" $\times$ "cue" $(F=0.094, P=0.963)$, "cue" $\times$ "flanker" $(F=0.603, P=$ $0.728)$, or "group" $\times$ "cue" $\times$ "flanker" $(F=0.045, P=$ 1.000). RTs were significantly longer in MwoA patients than in controls $(P<0.001)$. After Bonferroni correction, a significant difference was detected among flanker types

Table I Demographic Variables and Clinical Characteristics of Study Participants

\begin{tabular}{|c|c|c|c|c|}
\hline Characteristic & MwoA Patients $(n=44)$ & Healthy Controls $(n=20)$ & $\mathrm{T} / \chi^{2} / Z$ & $P$ value \\
\hline Age (years, mean $\pm S D$ ) & $41.55 \pm 12.11$ & $38.95 \pm 11.88$ & 0.799 & 0.427 \\
\hline Gender (n, female/male) & $40 / 4$ & $15 / 5$ & 1.714 & 0.191 \\
\hline Literacy (years;median, IQR) & $12(9,12)$ & $12(9,16)$ & -1.522 & 0.128 \\
\hline Migraine history (years, mean $\pm S D$ ) & $17.36 \pm 10.55$ & NA & NA & NA \\
\hline Headache days per month (days; median, IQR) & $9(4,20)$ & NA & NA & NA \\
\hline Pain intensity (score; median, IQR) & $7.5(7.00,8.38)$ & NA & NA & NA \\
\hline MSQ (score, mean \pm SD) & $40.91 \pm 9.74$ & NA & NA & NA \\
\hline SAS (score, mean \pm SD) & $43.70 \pm 10.10$ & $36.60 \pm 7.51$ & 2.807 & $0.007^{*}$ \\
\hline SDS (score, mean $\pm S D$ ) & $47.02 \pm 9.97$ & $37.45 \pm 10.49$ & 3.504 & $0.001 *$ \\
\hline
\end{tabular}

Notes: $* \mathrm{P}<0.05$ was considered statistically significant.

Abbreviations: MwoA, migraine without aura; MSQ, Migraine-Specific Quality of Life Questionnaire; SAS, Self Rating Anxiety Scale; SDS, Self-Rating Depression Scale; SD, standard deviation; IQR, interquartile range. 
Table 2 Mean RT and Accuracy Under Each Condition for Migraineurs and Controls

\begin{tabular}{|c|c|c|c|c|c|c|}
\hline \multirow[t]{3}{*}{ Flanker Type } & \multicolumn{6}{|c|}{ Cue Type } \\
\hline & \multicolumn{2}{|c|}{ Congruent } & \multicolumn{2}{|c|}{ Incongruent } & \multicolumn{2}{|c|}{ Neutral } \\
\hline & MwoA & $\mathrm{HC}$ & MwoA & $\mathrm{HC}$ & MwoA & $\mathrm{HC}$ \\
\hline \multicolumn{7}{|l|}{ Mean RT (ms) } \\
\hline No cue & $700.09 \pm 87.22$ & $628.45 \pm 124.97$ & $800.07 \pm 89.67$ & $699.75 \pm 140.40$ & $625.64 \pm 82.78$ & $593.90 \pm 126.58$ \\
\hline Center cue & $666.98 \pm 91.95$ & $591.90 \pm 140.55$ & $794.48 \pm 84.46$ & $690.60 \pm 145.00$ & $589.20 \pm 80.93$ & $532.10 \pm 125.67$ \\
\hline Double cue & $662.48 \pm 97.20$ & $589.40 \pm 137.66$ & $792.59 \pm 90.29$ & $682.80 \pm 142.92$ & $577.48 \pm 77.96$ & $525.85 \pm 122.73$ \\
\hline Spatial cue & $634.32 \pm 97.55$ & $561.85 \pm 138.17$ & $751.36 \pm 102.52$ & $639.50 \pm 148.53$ & $563.11 \pm 82.46$ & $517.15 \pm 133.83$ \\
\hline \multicolumn{7}{|l|}{ Accuracy } \\
\hline No cue & $1.00 \pm 0.01$ & $1.00 \pm 0.01$ & $0.98 \pm 0.04$ & $0.99 \pm 0.03$ & $0.99 \pm 0.02$ & $1.00 \pm 0.01$ \\
\hline Center cue & $0.99 \pm 0.02$ & $0.99 \pm 0.02$ & $0.97 \pm 0.05$ & $0.98 \pm 0.05$ & $1.00 \pm 0.0 \mathrm{I}$ & $1.00 \pm 0.01$ \\
\hline Double cue & $1.00 \pm 0.01$ & $1.00 \pm 0.00$ & $0.97 \pm 0.04$ & $0.97 \pm 0.04$ & $0.99 \pm 0.02$ & $0.99 \pm 0.02$ \\
\hline Spatial cue & $1.00 \pm 0.01$ & $1.00 \pm 0.01$ & $0.98 \pm 0.03$ & $0.99 \pm 0.20$ & $1.00 \pm 0.01$ & $1.00 \pm 0.10$ \\
\hline
\end{tabular}

Note: Data were shown as mean ( \pm standard deviation).

Abbreviations: RT, reaction time; MwoA, migraine without aura; HC, healthy controls.

$(P<0.001)$ in each group. RTs in the incongruent condition were longer than in the congruent or neutral condition for each group $(P<0.007)$.

ANOVA for accuracy revealed the significant main effect of flanker type $(F=45.60, P<0.001)$. No significant main effect of group $(F=0.225, P=0.635)$ or cue condition $(F=$
2.559, $P=0.054$ ), and no significant interactions among group, cue condition, and flanker type were detected.

\section{Network Efficiency Among Groups}

The efficiency of attention networks in ANT for each group is shown in Table 3. There were significant

Table 3 Uncorrected Raw Scores on the Attention Scale and RTs of the Three Attention Networks Between Migraineurs and Controls

\begin{tabular}{|c|c|c|c|c|}
\hline Parameters & MwoA $(n=44)$ & $H C(n=20)$ & $\mathrm{T} / \chi^{2} / Z$ & $P$ value \\
\hline \multicolumn{5}{|l|}{ Trail-Making Test (TMT) } \\
\hline TMT-A (s; median, IQR) & $33.76(22.76,40.95)$ & $23.83(\mid 3.34,31.50)$ & -2.904 & $<0.001 *$ \\
\hline TMT-B (s; median, IQR) & $53.11(43.79,65.63)$ & $35.23(29.30,48.79)$ & -4.012 & $<0.001 *$ \\
\hline TMT-d (s; median, IQR) & $23.29(14.94,33.64)$ & $12.05(6.09,18.85)$ & -3.056 & $0.002^{*}$ \\
\hline \multicolumn{5}{|l|}{ Digit Span Test (DST) } \\
\hline DST-f (n; median, IQR) & $8(7,8.75)$ & $9(8,10)$ & -3.894 & $<0.001 *$ \\
\hline DST-b (n; median, IQR) & $5(4,6)$ & $7.5(6,8)$ & -3.586 & $<0.00 I^{*}$ \\
\hline Digit Span Total (n, mean \pm SD) & $12.68 \pm 3.06$ & $16.05 \pm 2.01$ & -4.496 & $<0.00 I^{*}$ \\
\hline \multicolumn{5}{|l|}{ Stroop Test } \\
\hline Word Reading (n,mean $\pm S D)$ & $96.09 \pm 22.94$ & $102.95 \pm 17.11$ & -1.193 & 0.238 \\
\hline Color Naming $(n$, mean $\pm S D)$ & $63.4 I \pm 14.30$ & $65.10 \pm 11.55$ & -0.464 & 0.644 \\
\hline Color Word $(\mathrm{n}$, mean $\pm S \mathrm{~S})$ & $38.52 \pm 8.51$ & $41.80 \pm 8.32$ & -1.437 & 0.156 \\
\hline Interference Index (mean $\pm S D)$ & $0.55 \pm 7.37$ & $2.18 \pm 6.15$ & -0.860 & 0.393 \\
\hline \multicolumn{5}{|l|}{ Attention network test } \\
\hline Altering (ms, mean $\pm S D)$ & $29.18 \pm 20.99$ & $38.75 \pm 14.79$ & -1.838 & 0.071 \\
\hline Orienting (ms, mean $\pm \mathrm{SD}$ ) & $32.77 \pm 21.87$ & $35.25 \pm 13.653$ & -0.551 & 0.584 \\
\hline Executive control (ms, mean $\pm S D$ ) & $120.25 \pm 45.53$ & $87.16 \pm 31.89$ & 2.872 & $0.006 *$ \\
\hline Accuracy $($ mean $\pm S D)$ & $0.99 \pm 0.01$ & $0.99 \pm 0.01$ & -0.168 & 0.867 \\
\hline Overall RT (ms, mean $\pm S D)$ & $677.75 \pm 85.17$ & $602.10 \pm 134.12$ & 2.319 & $0.028 *$ \\
\hline
\end{tabular}

Note: $* \mathrm{P}<0.05$ was considered statistically significant.

Abbreviations: MwoA, migraine without aura; HC, healthy controls; IQR, interquartile range; SD, standard deviation. 
differences between groups on raw scores in the efficiency of the executive network $(P=0.006)$ and the overall mean RT $(P=0.028)$. No significant differences were found between groups on the alerting network $(P=0.071)$, orienting network $(P=0.584)$, and the overall accuracy $(P=0.867)$. Multiple linear regression adjusted for age, gender, literacy, and SAS, SDS showed that the efficiency of the executive network $(P=0.009)$ and the overall mean RT $(P=0.029)$ were independently associated with the diagnosis of MwoA (Table 4).

\section{Performance on the Attention Scale}

The scores on the Digit Symbol test, Stroop tests, and Trail-Making test are shown in Table 3. Significant group differences were found in the raw scores of TMT-A $(P<$ $0.001)$, TMT-B $(P<0.001)$, TMT-d $(P=0.002)$, DST-f $(P<0.001)$, DST-b $(P<0.001)$ and DS total $(P<0.001)$. There were no significant differences in the scores of the Stroop tests between the case and control groups. Multiple regression analysis adjusted for age, gender, literacy, and SAS, SDS revealed that the diagnosis of MwoA was independently correlated with the scores of $\log$ (TMT-d) $(P=0.011)$ and DS Total $(P=0.001)$, as shown in Table 4 .

Table 4 Multiple Regression Analysis to Assess the Role of Migraine without Aura in Attention Performances

\begin{tabular}{|l|c|c|c|}
\hline Dependent variable & $\begin{array}{c}\text { Adjusted } \\
\text { R Square }\end{array}$ & $\begin{array}{c}\text { MwoA (B) } \\
\text { (Difference } \\
\text { to Controls) }\end{array}$ & P value \\
\hline Attention network test & 0.134 & -9.828 & 0.089 \\
$\quad$ Altering & 0.023 & 4.012 & 0.507 \\
Orienting & 0.113 & 35.931 & $0.009^{*}$ \\
Executive control & 0.002 & 0.136 & 0.331 \\
SQRT (max+I-Accuracy) & 0.345 & 60.522 & $0.029 *$ \\
Overall RT & & & \\
\hline Trail-Making Test & 0.183 & 0.265 & $0.01 I^{*}$ \\
Log (TMT-d) & & & \\
\hline Digit Span Test & 0.522 & -2.453 & $0.00 I^{*}$ \\
Digit Span Total & & & \\
\hline Stroop Test & 0.035 & -2.591 & 0.693 \\
Word reading & 0.140 & 0.893 & 0.818 \\
Color naming & 0.230 & -2.584 & 0.271 \\
Color word & 0.078 & -2.531 & 0.231 \\
\hline Interference index & & & \\
\hline
\end{tabular}

Note: $* \mathrm{P}<0.05$ was considered statistically significant. Independent variables included in each multiple regression analysis were migraine without aura, gender, age, literacy, SAS, SDS. Adjusted R square represents the proportion of variance on each test score that is explained by the model. Abbreviation: B, unstandardized coefficient.

\section{Correlations Between Attention}

\section{Performances and Headache}

\section{Characteristics}

As shown in Table 5, multivariate linear regression analyses did not identify a correlation between the results of executive function (such as the efficiency of executive control, Log (TMT-d) and DS Total) and headache characteristics. We only found that the length of migraine history was independently positively correlated with the overall mean RT $(P=0.008)$.

\section{Discussion}

Attention plays a core role in human information processing. ${ }^{15}$ Our study revealed that migraineurs without aura show poorer attention performance than healthy subjects during the interictal period, evidenced by the ANT, DST, and TMT. Previous researches produced inconsistent results about attention deficits in the headache-free period, owing to the discrepancies in migraineurs' characteristics, the definition of attention, and the application of qualitatively varied instruments to measure attention. ${ }^{4,10}$ In order to ensure the homogeneity of the study subjects, the included participants were matched for age, gender, education. Raw data were corrected, controlling by the possible confounders by the regression analysis. We adopt the ANT, which can detect three independent networks of attention and have good validity. ${ }^{23,24}$ Our results from the ANT showed that the reaction time of the executive network and the overall mean reaction time in MwoA were longer compared with healthy controls, whereas no differences were detected in the efficiency of the alerting and orienting network. This finding indicated that migraineurs exhibited poorer attention performance on executive function than controls during headache-free periods, and we may speculate that the overall attention impairment was specifically reflected in the executive control network. In addition, the data from the TMT, DST (mainly included TMT-B, TMT-d, DST-b, and Digit Span Total) further verified the results that patients in MwoA made slower responses in executive function, which was consistent with previous studies reported by Ferreira et $\mathrm{al}^{25}$ and Vallesi et al. $^{26}$

Executive control, as one of three independent attention networks, is defined as monitoring and resolving conflict among responses and overcoming habitual actions, ${ }^{23}$ involving working memory, attention shift, cognitive flexibility and so on. Cognitive disorder, particularly 
Table 5 Linear Multivariate Regression Analyses Between Clinical Characteristics and Attention Performance in Migraineurs without Aura

\begin{tabular}{|c|c|c|c|c|c|c|c|c|c|c|c|c|}
\hline \multirow[t]{2}{*}{ Variable } & \multicolumn{3}{|c|}{ Overall Mean RT (ms) } & \multicolumn{3}{|c|}{ Executive Control (ms) } & \multicolumn{3}{|c|}{$\log (T M T-d)$} & \multicolumn{3}{|c|}{ DST Total } \\
\hline & $\beta$ & SE & $\mathbf{P}$ & $\beta$ & SE & $\mathbf{P}$ & $\beta$ & SE & $\mathbf{P}$ & $\beta$ & SE & $\mathbf{P}$ \\
\hline \multicolumn{13}{|l|}{ Univariate linear regression analyses } \\
\hline Migraine history & 3.763 & 1.103 & 0.001 & -0.866 & 0.653 & 0.192 & 0.011 & 0.005 & 0.944 & -0.248 & 0.043 & 0.104 \\
\hline Attack days per month & 3.577 & 1.628 & 0.034 & -0.234 & 0.918 & 0.800 & 0.007 & 0.006 & 0.265 & -0.015 & 0.062 & 0.808 \\
\hline Pain intensity & -10.69 & 7.665 & 0.170 & 6.741 & 4.060 & 0.104 & 0.093 & 0.029 & 0.549 & -0.288 & 0.269 & 0.058 \\
\hline MSQ & 1.324 & 1.334 & 0.327 & 0.525 & 0.112 & 0.468 & 0.042 & 0.005 & 0.787 & -0.013 & 0.048 & 0.931 \\
\hline SAS & 0.691 & 1.296 & 0.597 & 0.564 & 0.690 & 0.418 & -0.004 & 0.005 & 0.977 & -0.530 & 0.047 & 0.599 \\
\hline SDS & 0.046 & 1.318 & 0.972 & 0.348 & 0.702 & 0.623 & 0.065 & 0.005 & 0.676 & -0.077 & 0.047 & 0.620 \\
\hline \multicolumn{13}{|l|}{ Multivariate linear regression analyses } \\
\hline Migraine history & 3.252 & 1.173 & $0.008^{*}$ & NA & NA & NA & NA & NA & NA & NA & NA & NA \\
\hline Attack days per month & 1.981 & 1.618 & 0.228 & NA & NA & NA & NA & NA & NA & NA & NA & NA \\
\hline Pain intensity & NA & NA & NA & NA & NA & NA & NA & NA & NA & -0.288 & 0.269 & 0.058 \\
\hline
\end{tabular}

Note: $* \mathrm{P}<0.05$ was considered statistically significant.

Abbreviations: TMT, Trail-Making Test; DST, Digit Span test; MSQ, Migraine-Specific Quality of Life Questionnaire; SAS, Self-Rating Anxiety Scale; SDS, Self-Rating Depression Scale.

impairment in executive function, could contribute to migraine attack-related disability. ${ }^{10}$ It was generally considered that migraine-related manifestations disappeared in the interictal period. However, evidence from neurophysiological and neuroimaging studies revealed that interictal differences differed in cortical excitability and pain processing from controls. ${ }^{10}$ Huang et $\mathrm{al}^{27}$ had reported that the functional connectivity between the left red nucleus and ipsilateral middle frontal gyrus, which were involved in the pain processing, modulation, and cognitive evaluation, was significantly decreased in migraineurs without aura during interictal periods. Based on the detected reduced functional connectivity within the frontoparietal networks, MwoA patients could experience some degree of difficulty in daily life involving complex executive processes. ${ }^{28}$ Posner and Petersen ${ }^{14}$ proposed that the sources of the executive control network form a specific system of anatomical areas, which involved the midline frontal areas, lateral prefrontal cortex, ventral- and dorsolateral prefrontal cortex, insula, ${ }^{29,30}$ and cerebellum. ${ }^{31}$ Kruit et al reported an increased risk of subclinical stroke in the cerebellum, a region of the posterior circulation, in migraineurs. ${ }^{32}$ A study based on a high-field MRI scanner and voxel-based morphometry had shown that reduced gray matter density in $\mathrm{T} 2$ visible areas of the brain, mainly in the frontal and temporal lobes, was detected in migraine patients during a headache-free period. ${ }^{33}$ These structural and functional changes in migraineurs' brains may provide a potential neurobiological mechanism to explain the impairment of executive function. In addition, the executive control of attention networks is a neurological function that depends not only on the independent anatomic structure but also on some neurotransmitters such as dopamine. ${ }^{34}$ Dopamine dysfunction is hypothesized to be a causal factor in migraine pathogenesis, and subjects with midlife migraine were related to a higher prevalence of late-life Parkinsonism symptoms, Parkinsonism disease, and restless legs syndrome (RLS). ${ }^{35}$

In order to identify potential predictors of attention impairment in subjects with MwoA, we evaluated the correlation between attention performance and clinical characteristics in migraineurs. Previous studies had found that constant suffering of mood disorders was clearly a important factor affecting cognition. ${ }^{36}$ However, in our study, all analyses adjusted for demographics, headache characters, and psychiatric symptoms as covariates found no association between anxiety, depression, and impaired attention, which was consistent with results reported in some previous studies. ${ }^{13,37}$ Furthermore, we only found a positive correlation between the years of migraine history and overall mean reaction time. A longer migraine history could signify higher age, leading by itself to longer reaction times. However, it is worth mentioning a recent study showing that migraine and non-migraine headache are not associated with increasing risk of dementia or cognitive decline at an older age although subjects with migraine have more cognitive complaints. ${ }^{38}$ However, we did not find a correlation between the measures of migraine severity and efficiency of executive function. Previous studies ${ }^{39-41}$ have reported similar findings in 
cognition assessments, in which cognitive performance was unrelated to the length of headache history, severity, and duration of migraine attacks. These findings need to be confirmed by clinical studies with a large sample for longterm follow-up. Meanwhile, further research is needed to investigate whether other features of headache, such as the side or type of pain or associated symptoms, are associated with executive dysfunction. Recent studies showed that polymorphisms at genes COMTVal158Met, DRD2/ ANKK1, DRD4, DBH, and CHRNA4 were associated with executive function. ${ }^{42}$ These genetic polymorphisms were also reported to be related to the susceptibility for migraine, although evidences for such an association were inconsistent. $^{43-45}$ These findings may indicate that migraine and executive dysfunction share a common risk factor influences, rather than one leading to the other. Further studies could investigate the genetic susceptibility of migraineurs to executive function impairment.

At this point, some limitations of our study should be considered. Firstly, the sample size in our study was small, which may be related to the negative result of the stroop tests between patients and controls. Although these are not statistically significant, the direct scores for stroop test show more completions among the MwoA group than in the control group. Meanwhile, considering the lack of sample size, we did not conduct further subgroup analysis based on the monthly onset time of MwoA. Secondly, as a cross-sectional design study, we could not evaluate the progress of cognitive decline in these patients. Thirdly, the study is clinic-based, where patients were drawn from a tertiary, first class hospitals with low attendance rates for patients with mild headache. Therefore, the current findings must be viewed with caution as they do not necessarily reflect the cognitive status of patients in the community or individuals with milder forms of migraine. Further study with large sample size is needed to evaluate the changes of cognitive performance with disease progress. The strong aspect of this study is that the inclusion of only migraine who do not practice prophylactic treatment increasing the possibility of affecting test performance, which increased the accuracy of the study.

\section{Conclusion}

In summary, our results show that adult patients with MwoA exhibit selective impairment in executive function during the headache-free period. Anxiety, depression, and measures of migraine severity did not influence the executive performance in MwoA sufferers. Executive dysfunction identified in migraine patients implies the nature of cortical dysfunction in migraine, which provide a new perspective to understand the pathophysiology of migraine. Executive function impairment may contribute to migraine-related disability and should be considered as a target of acute and preventive migraine treatment. We suggest that migraineurs should undergo a neuropsychological screening to assess attention, in order to find a more appropriate therapeutic management.

\section{Acknowledgments}

We wish to thank the control subjects and the patients who participated in this experiment.

\section{Author Contributions}

TW and ZCS conceived and designed the work. TW, CC, $\mathrm{XD}, \mathrm{PG}, \mathrm{KYC}$, and HYX participated in acquired the data. TW, CC, XD, and PG performed the statistical analysis under the supervision of ZCS and QW. CC wrote the original draft, while $\mathrm{XD}, \mathrm{TW}$, and QW revised the draft. All authors made a significant contribution to the work reported, whether that is in the conception, study design, execution, acquisition of data, analysis and interpretation, or in all these areas; took part in drafting, revising or critically reviewing the article; gave final approval of the version to be published; have agreed on the journal to which the article has been submitted; and agree to be accountable for all aspects of the work.

\section{Disclosure}

The authors declare that they have no competing interests.

\section{References}

1. Tolner EA, Chen SP, Eikermann-Haerter K. Current understanding of cortical structure and function in migraine. Cephalalgia. 2019;39 (13):1683-1699. doi:10.1177/0333102419840643

2. Goadsby PJ, Holland PR, Martins-Oliveira M, Hoffmann J, Schankin C, Akerman S. Pathophysiology of migraine: a disorder of sensory processing. Physiol Rev. 2017;97(2):553-622. doi:10.1152/ physrev.00034.2015

3. Stovner LJ, Nichols E, Steiner TJ; Collaborators GBDH. Global, regional, and national burden of migraine and tension-type headache, 1990-2016: a systematic analysis for the global burden of disease study 2016. Lancet Neurol. 2018;17(11):954-976. doi:10.1016/ S1474-4422(18)30322-3

4. Gil-Gouveia R, Martins IP. Cognition and cognitive impairment in migraine. Curr Pain Headache Rep. 2019;23(11):84. doi:10.1007/ s11916-019-0824-7

5. Calandre EP, Bembibre J, Arnedo ML, Becerra D. Cognitive disturbances and regional cerebral blood flow abnormalities in migraine patients: their relationship with the clinical manifestations of the illness. Cephalalgia. 2002;22(4):291-302. doi:10.1046/j.1468-2982.20 02.00370.x 
6. de Araujo CM, Barbosa IG, Lemos SMA, Domingues RB, Teixeira AL. Cognitive impairment in migraine: a systematic review. Dement Neuropsychol. 2012;6(2):74-79. doi:10.1590/S19 80-57642012DN06020002

7. Schmitz N, Arkink EB, Mulder M, et al. Frontal lobe structure and executive function in migraine patients. Neurosci Lett. 2008;440 (2):92-96. doi:10.1016/j.neulet.2008.05.033

8. Suhr JA, Seng EK. Neuropsychological functioning in migraine: clinical and research implications. Cephalalgia. 2012;32(1):39-54. doi: $10.1177 / 0333102411430265$

9. Coull JT. Neural correlates of attention and arousal: insights from electrophysiology, functional neuroimaging and psychopharmacology. Prog Neurobiol. 1998;55(4):343-361. doi:10.1016/S0301-0082(98)00011-2

10. Vuralli D, Ayata C, Bolay H. Cognitive dysfunction and migraine. J Headache Pain. 2018;19(1):1-4.

11. Gil-Gouveia R, Oliveira AG, Martins IP. Subjective cognitive symptoms during a migraine attack: a prospective study of a clinic-based sample. Pain Physician. 2016;19(1):E137-E150. doi:10.36076/ppj/ 2016.19.E137

12. Huang LF, Dong HJ, Wang X, Wang Y, Xiao ZM. Duration and frequency of migraines affect cognitive function: evidence from neuropsychological tests and event-related potentials. J Headache Pain. 2017;18(1):1-7.

13. Camarda C, Monastero R, Pipia C, Recca D, Camarda R. Interictal executive dysfunction in migraineurs without aura: relationship with duration and intensity of attacks. Cephalalgia. 2007;27(10):10 94-1100. doi:10.1111/j.1468-2982.2007.01394.x

14. Posner MI, Petersen SE. The attention system of the human brain. Annu Rev Neurosci. 1990;13(1):25-42. doi:10.1146/annurev. ne.13.030190.000325

15. Fan J, McCandliss BD, Sommer T, Raz A, Posner MI. Testing the efficiency and independence of attentional networks. J Cognitive Neurosci. 2002;14(3):340-347. doi:10.1162/089892902317361886

16. Pauletti C, Mannarelli D, Locuratolo N, et al. Attention in Parkinson's disease with fatigue: evidence from the attention network test. $J$ Neural Transm. 2017;124(3):335-345. doi:10.1007/s00702-016-1637-z

17. Johnson KA, Robertson IH, Barry E, et al. Impaired conflict resolution and alerting in children with ADHD: evidence from the Attention Network Task (ANT). J Child Psychol Psychiatry. 2008;49(12):1339-1347. doi:10.1111/j.1469-7610.2008.01936.x

18. Urbanek C, Weinges-Evers N, Bellmann-Strobl J, et al. Attention network test reveals alerting network dysfunction in multiple sclerosis. Mult Scler. 2010;16(1):93-99. doi:10.1177/1352458509350308

19. Headache Classification Committee of the International Headache S. The international classification of headache disorders, 3rd edition (beta version). Cephalalgia. 2013;33(9):629-808. doi:10.1177/ 0333102413485658

20. Giovagnoli AR, Del Pesce M, Mascheroni S, Simoncelli M, Laiacona M, Capitani E. Trail making test: normative values from 287 normal adult controls. Ital J Neurol Sci. 1996;17(4):305-309. doi:10.1007/BF01997792

21. Lopez E, Steiner AJ, Hardy DJ, IsHak WW, Anderson WB. Discrepancies between bilinguals' performance on the Spanish and English versions of the WAIS digit span task: cross-cultural implications. Appl Neuropsychol. 2016;23(5):343-352. doi:10.1080/23279095.2015.1074577

22. Scarpina F, Tagini S. The stroop color and word test. Front Psychol. 2017;8:557. doi:10.3389/fpsyg.2017.00557

23. Posner MI. Measuring alertness. Ann N Y Acad Sci. 2008;1129 (1):193-199. doi:10.1196/annals.1417.011

24. Chun MM, Golomb JD, Turk-Browne NB. A taxonomy of external and internal attention. Annu Rev Psychol. 2011;62(1):73-101. doi:10.1146/annurev.psych.093008.100427

25. Ferreira KS, Teixeira CT, Cafaro C, et al. Chronic migraine patients show cognitive impairment in an extended neuropsychological assessment. Arq Neuropsiquiat. 2018;76(9):582-587. doi:10.1590/ 0004-282x20180085
26. Vallesi A. On the utility of the trail making test in migraine with and without aura: a meta-analysis. J Headache Pain. 2020;21(1). doi:10.1186/s10194-020-01137-y

27. Huang XB, Zhang D, Chen YC, et al. Altered functional connectivity of the red nucleus and substantia nigra in migraine without aura. J Headache Pain. 2019;20(1). doi:10.1186/s10194-019-1058-0.

28. Russo A, Tessitore A, Giordano A, et al. Executive resting-state network connectivity in migraine without aura. Cephalalgia. 2012;32(14):1041-1048. doi:10.1177/0333102412457089

29. Aron AR, Robbins TW, Poldrack RA. Inhibition and the right inferior frontal cortex. Trends Cogn Sci. 2004;8(4):170-177. doi:10.1016/j. tics.2004.02.010

30. Wager TD, Jonides J, Reading S. Neuroimaging studies of shifting attention: a meta-analysis. Neuroimage. 2004;22(4):1679-1693. doi:10.1016/j.neuroimage.2004.03.052

31. Heyder K, Suchan B, Daum I. Cortico-subcortical contributions to executive control. Acta Psychol. 2004;115(2-3):271-289. doi:10.10 16/j.actpsy.2003.12.010

32. Kruit MC, van Buchem MA, Hofman PAM, et al. Migraine as a risk factor for subclinical brain lesions. J Am Med Assoc. 2004;291 (4):427-434. doi:10.1001/jama.291.4.427

33. Rocca MA, Ceccarelli A, Falini A, et al. Brain gray matter changes in migraine patients with T2-visible lesions - A 3-T MRI study. Stroke. 2006;37(7):1765-1770. doi:10.1161/01.STR.0000226589.00599.4d

34. Petersen SE, Posner MI. The attention system of the human brain: 20 years after. Annu Rev Neurosci. 2012;35(1):73-89. doi:10.1146/ annurev-neuro-062111-150525

35. Scher AI, Ross GW, Sigurdsson S, et al. Midlife migraine and late-life parkinsonism AGES-reykjavik study. Neurology. 2014;83 (14):1246-1252. doi:10.1212/WNL.0000000000000840

36. Stillman AN, Rowe KC, Arndt S, Moser DJ. Anxious symptoms and cognitive function in non-demented older adults: an inverse relationship. Int J Geriatr Psych. 2012;27(8):792-798. doi:10.1002/gps.2785

37. Lo Buono V, Bonanno L, Corallo F, et al. Cognitive functions and psychological symptoms in migraine: a study on patients with and without aura. Int J Neurosci. 2019;129(6):588-592. doi:10.1080/ 00207454.2018.1554658

38. Martins IP, Maruta C, Alves PN, et al. Cognitive aging in migraine sufferers is associated with more subjective complaints but similar age-related decline: a 5-year longitudinal study. J Headache Pain. 2020;21(1):31. doi:10.1186/s10194-020-01100-x

39. Mulder EJCM, Linssen WHJP, Passchier J, Orlebeke JF, de Geus EJC. Interictal and postictal cognitive changes in migraine. Cephalalgia. 1999;19(6):557-565.

40. Hooker WD, Raskin NH. Neuropsychologic alterations in classic and common migraine. Arch Neurol. 1986;43(7):709-712. doi:10.1001/ archneur.1986.00520070065020

41. Bell BD, Primeau M, Sweet JJ, Lofland KR. Neuropsychological functioning in migraine headache, nonheadache chronic pain, and mild traumatic brain injury patients. Arch Clin Neuropsychol. 1999;14(4):389-399. doi:10.1093/arclin/14.4.389

42. Logue SF, Gould TJ. The neural and genetic basis of executive function: attention, cognitive flexibility, and response inhibition. Pharmacol Biochem Behav. 2014;123:45-54. doi:10.1016/j.pbb.2013.08.007

43. Mochi M, Cevoli S, Cortelli P, et al. A genetic association study of migraine with dopamine receptor 4, dopamine transporter and dopamine-beta-hydroxylase genes. Neurol Sci. 2003;23(6):301-305. doi:10.1007/s100720300005

44. Liu JX, Lan L, Mu JY, et al. Genetic contribution of catechol-O-methyltransferase in hippocampal structural and functional changes of female migraine sufferers. Hum Brain Mapp. 2015;36(5):1782-1795. doi:10.1002/hbm.22737

45. Ghosh J, Pradhan S, Mittal B. Identification of a novel ANKK1 and other dopaminergic (DRD2 and $\mathrm{DBH}$ ) gene variants in migraine susceptibility. Neuromol Med. 2013;15(1):61-73. doi:10.1007/s12 017-012-8195-9 


\section{Publish your work in this journal}

The Journal of Pain Research is an international, peer reviewed, open access, online journal that welcomes laboratory and clinical findings in the fields of pain research and the prevention and management of pain. Original research, reviews, symposium reports, hypothesis formation and commentaries are all considered for publication. The manuscrip management system is completely online and includes a very quick and fair peer-review system, which is all easy to use. Visit http:// www.dovepress.com/testimonials.php to read real quotes from published authors. 